\title{
On Creative Thinking in Advertising Design Based on Modern
}

\author{
Elements
}

\author{
Xiaorong Yuan \\ Guangxi Arts University, Nanning, 530022, China
}

\begin{abstract}
Advertising designers shall carry out creative advertising design activities based on modern elements, in order to change people's fixed views on a certain commodity and make them have a strong desire to buy. In this sense, creative thinking in advertising design is an art creation activity and is the soul of excellent and successful advertising design. Therefore, this paper discusses deeply about creative thinking in advertising design from the perspective of modern elements, and believes that advertising designers shall analyze advertising psychological needs of modern people and express creativity of advertising design from multiple perspectives to make advertising and publicity, communication, and promotion functions of advertising design more prominent.
\end{abstract}

Key words: modern elements, advertising design, creative thinking

\section{Topic background}

With continuous development of China's market economy, Chinese enterprises face increasingly fierce international competition. Under such a feast of international cultural and economic exchanges, it is a key to achieve rapid and stable development in Chinese advertising industry, as advertising relates directly to product sales in a sense and finally affects enterprise profitability. Therefore, to cater to such a development context, advertising design shall seek better creative thinking from modern elements, improve technical content of enterprise products constantly, enhance product quality and service level, and allow some foreign enterprises to join advertising design market if necessary, so that Chinese advertising design would have more international creative thinking, better participate in international competition, gain better international development opportunities, expand its market space, and finally promote rapid, stable and prolonged development of Chinese advertising industry.

\section{Creative thinking in advertising design}

With enhancing economic strength and increasingly fierce market competition in current China, a lot of commercial competitions have changed from traditional product competition to competition of intelligence, and many enterprises are vigorously involved in advertising publicity, making commodity sales of many enterprises become a media war of advertising publicity. Actually, in modern context, struggle in advertising industry gradually rises to struggle of advertising creativity, which becomes one of the most popular phrases in current advertising industry with meanings mainly including creation, establishment and formation etc. Thus, creative thinking in advertising design refers to an artistic conception behavior between advertising planning and specific expression \& production. It requires 
advertising designers to design and create meticulously according to connotation of actual advertising themes, combine and reconstruct all creation materials creatively, in order to make advertising works more lively and vivid. In other words, it is a process of imagery design of themes of advertising works. Here, advertising themes mean concepts to be expressed with specific purposes, and cannot be seen or touched, but indeed exist in advertised commodities, and can only be reflected with the help of visible things. Thus, creative thinking in advertising design is an artistic means to reproduce advertising themes and an artistic channel to realize effective communication between advertising themes and the masses.

So, in current society, a lot of advertising spreads over every corner of the world However, some advertising is extremely ordinary, while some is difficult to leave a deep impression even with unique looks. This is because creative thinking of such advertising design isn't in line with aesthetic psychological needs of modern people, and some advertising designers have limited technical level and create a lot of rubbish advertising works, which cannot have due advertising effects or cannot realize advertising designers' expectation of advertising design. Thus, by analyzing from this perspective, the fundamental influencing factor of the success of advertising design is whether creative thinking is unique. Advertising works without creative thinking are unsuccessful and cannot have effective information transmission. Advertising design shall gain development driving forces from creative thinking, so as to make advertising themes more artistic and expressive, contain more ideas, thoughts and artistic conception, and realize a perfect unity of being both strategic and novel. Here, creative thinking of advertising design can be summarized as follows: creative thinking is unique expression and expressing method of ideas with fundamental principle of information transmission, and can better explain advertising themes through unique pictures and images etc, stimulate the audience's interests and their desire to buy and buying motivators, and thus give real play to promotion function of advertising.

\section{Several basic methods of creative thinking in advertising design}

Advertising design itself is an artistic activity and requires designers to give full play to their imagination and creativity in creation. Generally speaking, creative thinking in advertising design can be realized by the following methods:

(1) Lateral thinking method. This method is first put forward by Dr. De Bono, who is an ecological physiologist of University of Cambridge, in his The World of Lateral Thinking, and gradually becomes a scientific thinking method widely recognized and applied in other fields. It requires advertising designers to observe and conceive from multiple perspectives to make advertising themes more diversified. To implement this creative thinking, advertising designers shall break traditional concepts constantly, try new things, attach importance to channels of new concepts, consider about more possibilities, and pursue richness and opportunities. Essentially, lateral thinking method is actually to mobilize designers' thinking for various aspects of association, including emotion, narration, property and state etc. It enables advertising designers to discover maximum advertising creativity, show the most abundant advertising implication, and improve their thinking ability.

(2) Analogy thinking method. If applied to advertising design, this method will make element projection more characterized and personalized in advertising themes, guide the masses to go deeper into advertising themes and have emotional resonance. Analogy method 
in advertising design is a simple and lifelike creative technique with amiable emotional implication, and is easy to arouse spiritual resonance of the masses, stimulate their imagination and creativity and form a benign and interactive design context between creators and the masses. Therefore, advertising designers make these commodities gain certain personalized effects through this method, and help achieve emotional communication and exchanges between products and the masses.

(3) Comparative association method. In the objective world, the law of unity of opposites is almost universal. The way people think about things is also comparative. Thus, creative thinking in advertising design is obviously inseparable from comparative association method, which allows people to seize some important clues and information through comparison of ways of thinking. In fact, people could get strong psychological or physical cues through comparison and highlight features of some things more distinctively. Then, some advertising designers make full use of this trait of comparison, and compare strongly some objects in terms of both graphs and colors, make the masses have strong visual impact and gain strong spiritual interaction, and thus impress people deeply and lastingly through advertising.

(4) Combination and replacement method. This method refers to a process of thinking activity that links different natures of things or functions according to specific needs, recombines and rearranges several elements, and finally creates new things or ideas. Thus, creative thinking in advertising design is basically the creation result of putting known materials together, and it is quite difficult to truly discover new materials or design. That is to say, advertising designers shall investigate and analyze advertising creation materials from multiple perspectives, recombine or replace these materials, generate new advertising meanings, and provide the masses with different advertising content. This method is advantageous in terms of creative thinking, and is the most efficient and convenient creation path, and is the easiest language to arouse resonance and communicate, and basically includes element recombination and replacement etc.

(5) Humorous creativity method. The most excellent character of human culture is a sense of humor, which is concentrated reflection of high human wisdom and is able to give artistic creation boundless glamour and strong appeal. Then, humorous creativity method is a method of creative thinking in advertising design, and it can give advertising works greater affinity and appeal. This is because the masses could understand the connotation of advertising in a relaxing and pleasant advertising atmosphere, change their attitude towards and recognition of advertised commodities consciously, and stimulate their desire to buy. Advertising designers could make advertising more amiable and lovely through these humorous design techniques, stimulate consumers' interest in commodities, and thus facilitate successful commodity sales.

\section{Specific application of creative thinking in advertising design based on modern elements}

In view of the importance and necessity of creative thinking in advertising design, we shall apply such creative thinking specifically based on modern elements, make advertising design show its value and significance, lead the masses to really gain more numerous and more accurate commodity information from advertising, and thus promote successful commodity sales and realize the fundamental purpose of advertising. Generally speaking, creative thinking can be applied to advertising design from the following aspects: 
(1) Imagery thinking shall be applied well to advertising design. As an abstract imagery way of thinking, imagery thinking is an important link of creative thinking in advertising design, and is a creative design process by thinking through a specific image. Long time ago, people had formed a point of view, i.e. visual art thinking is based on imagery thinking. When carrying out artistic creation, people would often come up with many specific images in mind, and then have artistic processing and creation of thinking with these images. Therefore, such imagery thinking is needed in creative thinking in advertising design to stimulate imagination and creativity of designers, and enables designers to reselect and rearrange creation materials through active thinking ability, form a new rational will and achieve harmonious unity of advertising design. That is to say, advertising designers could gain more beautiful artistic sensibility through imagination when facing a lot of things, carry out artistic processing based on abilities of the masses, realize imagery tacit understanding between imagination and receptivity of the masses, and thus make advertising design promote products or services in advertising successfully to the masses.

(2) Divergent thinking shall be applied well to advertising design. In modern advertising design, divergent thinking is also a very important element with characteristics of diffusivity, coverage and multi-direction, and it can guide designers to develop in a variety of directions and gain multiple ways of thinking and methods in creation. Essentially, divergent thinking is an illogic way of thinking that starts from a central problem and then converts with multiple ideas. It could enable designers to break through original mind-set and find out more novel creation design schemes according to existing design experience and theoretical knowledge foundation. Therefore, it hasn't a fixed creation mode, and presents to be more open and arbitrary, and thus is an unconventional way of thinking. Thus, divergent thinking can capture more creative materials for advertising design, promote innovative development of advertising design activities, and make people feel a new creative force to express creativity of advertising themes. Besides, reverse thinking shall be applied well to advertising design. Reverse thinking belongs to a very special way of thinking in creative thinking in modern advertising design, and is actually reverse expression of divergent thinking. It is also a very novel creative thinking in advertising design by which people start from conventional reverse thinking and advertising design shows different advertising effects. Moreover, it avoids long-term mind-set, allows people to gain different advertising effects through reverse thinking, and facilitates the play of advertising role.

(3) Inspiration thinking shall be applied well to advertising design. In advertising design, a climactic creation stage will occur in application of modern elements, and then designers' creation inspiration will be stimulated. In fact, inspiration thinking is also an indispensable form of creative psychological activities for advertising designers, an expression of designers' highly concentrated thinking during ideation and a creation psychology generated in a moment. Such inspiration is called sudden enlightenment in psychology because of temporary emotional relaxation or accidental enlightenment of events etc. Of course, inspiration is not an artistic means owned by everyone, as it requires learning of knowledge and accumulation of experience after long-run labor, good mental state and harmonious existence with external environment etc, and also requires designers' long-term thinking and dedicated exploration etc. Thus, inspiration is actually the result of diligent labor. However, it is instable and uncertain in terms of time, and is a guest without traces. Designers shall grasp inspiration 
firmly, get everything ready, change their inspiration thinking truly into practical artistic creation, and thus create advertising design works with combination of body and soul.

\section{Acknowledgments}

Foundation No. of this paper: [2010]10 No. 201010LX319, College of Art [2010] No. 254

\section{References:}

[1] Bo Guanxiu, Jia Ronglin. Creativity and Expression of Modern Graphic Design [M]. Beijing: China INTL Culture Press, 1993

[2] Han Jixian, Xuan Hongbo. Visual Communication Design of Graphic Advertising [J]. Journal of Inner Mongolia University for Nationalities, 2005 (02)

[3] Tian Zhimei, Yin Chaoyang. Integration of Traditional Chinese Cultural Elements with Modern Poster Design-An Example of Prize-winning Works in Tongxiang Chrysanthemum Festival [J]. Theory Monthly, 2014 (06)

[4] Wang Shouzhi. A History of Modern Design Worldwide [M]. Guangzhou: New Century Publishing House, 2001

[5] Zhang Juan. How to Train Students' Creative Thinking in Vocational College Advertising Design Teaching [J]. Art and Literature for the Masses, 2014 (14) 